Chronique des activités archéologiques de

\title{
Cimitero di Atella
}

Nouvelles recherches et valorisation d'un gisement du Paléolithique ancien en Méditerranée

Roxane Rocca, Daniele Aureli et Claudia Abruzzese

\section{Q OpenEdition}

\section{Journals}

Édition électronique

URL : http://journals.openedition.org/cefr/1910

DOI : $10.4000 /$ cefr. 1910

ISSN : 2282-5703

Éditeur

École française de Rome

\section{Référence électronique}

Roxane Rocca, Daniele Aureli et Claudia Abruzzese, "Cimitero di Atella », Chronique des activités archéologiques de l'École française de Rome [En ligne], Italie du Sud, mis en ligne le 09 avril 2018, consulté le 05 septembre 2019. URL : http://journals.openedition.org/cefr/1910 ; DOI : 10.4000/ cefr. 1910

Ce document a été généré automatiquement le 5 septembre 2019

(c) École française de Rome 


\title{
Cimitero di Atella
}

Nouvelles recherches et valorisation d'un gisement du Paléolithique ancien en Méditerranée

\author{
Roxane Rocca, Daniele Aureli et Claudia Abruzzese
}

\section{NOTE DE L'AUTEUR}

Les nouvelles recherches menées à Atella sont financées par l'École française de Rome dans le cadre de l'axe 2 du projet quinquennal (2017-2021) PALEO. Les recherches sur place sont soutenues par la Commune d'Atella et la région Basilicate. L'autorisation de fouille et d'étude du matériel nous est confiée par la Soprintendenza Archeologia Belle Arti e Paesaggio della Basilicata. Les cahiers de fouilles et les anciennes collections sont conservés au Museo archeologico nazionale del Melfese, et accessibles grâce à la bienveillance du Professeur E. Borzatti von Löwenstern. Les analyses et missions de terrains sont prises en charges par les institutions des chercheurs qui collaborent au projet : Jean-Jacques Bahain (UMR 7194 HNHP) ; Paolo Beneduce (Università degli Studi della Basilicata) ; Francesco Boschin (Università degli Studi di Siena) ; Paola Di Leo (CNRIMAA) ; Federico Di Rita (Università di Roma La Sapienza) ; Paolo Giannandrea (Università degli Studi della Basilicata); Salvatore Ivo Giano (Università degli Studi della Basilicata); Dario Gioia (CNR-IBAM) ; Donatella Magri (Università di Roma La Sapienza) ; Sebastien Nomade (UMR 8212, LSCE) ; Alison Pereira (UMR 7194 HNHP) ; Raffaele Sardella (Università di Roma La Sapienza); Marcello Schiattarella (Università degli Studi della Basilicata) ; Roberto Sulpizio (Università degli Studi di Bari Aldo Moro) ; Pierre Voinchet (UMR 7194 HNHP) ; Maurizio Zambaldi (Università degli studi di Trento).

\section{Introduction}

1 Les nouvelles recherches initiées en 2015 à Atella (Basilicate, Italie) visent à renouveler les hypothèses sur les premières dynamiques de peuplement en Méditerranée à travers l'étude de la structuration culturelle de l'espace dans la péninsule italienne. Malgré sa 
situation jugée périphérique au sein de l'Europe, l'Italie témoigne au cours du Paléolithique ancien (entre 1,4 et 0,3 millions d'années) d'une étonnante richesse en termes de quantité de données, de diversité des systèmes techniques, et est souvent précurseur des grandes innovations techniques.

2 Le peuplement paléolithique de l'Europe est généralement considéré comme le résultat de vagues successives venues d'Afrique par le Proche-Orient et l'Europe centrale ${ }^{1}$. Les deux premières vagues, attribuées au Paléolithique inférieur, seraient chacune caractérisées par un type d'outil spécifique : outils sur éclats pour la première $(1,4 \mathrm{Ma})$, et biface pour la deuxième $(0,7 \mathrm{Ma})$. Dans ce modèle, l'Italie comme l'Espagne ou l'Angleterre sont donc considérées comme zones périphériques, voire comme des culs-desac aux confins de l'Europe. Pourtant, c'est précisément dans ces trois régions que l'on retrouve les plus anciens sites ${ }^{2}$, alors que la région intermédiaire, entre le Proche-Orient et l'Est de la France, en est totalement dépourvue ${ }^{3}$.

Ce paradoxe est particulièrement frappant en ce qui concerne la péninsule italienne et notamment le Centre-Sud. S'il s'agit physiquement de l'un des territoires les plus retirés, c'est aussi là qu'émergent la plupart des grandes innovations techniques au cours du Paléolithique et que l'on retrouve un « concentré » de toute la diversité des expressions techniques présentes en Europe ${ }^{4}$. Ces éléments nous invitent donc à nous interroger sur le rôle de l'Italie dans le Paléolithique ancien en Méditerranée. Avons-nous affaire à une région d'innovation? À une zone refuge? Ou encore à une "plaque-tournante » où les idées pénètrent et refluent?

4 Les premiers sites ayant livré des bifaces en Europe apparaissent aux alentours de 700 000 ans en Espagne, en France et en Italie ${ }^{5}$. Deux de ces sites se trouvent en Basilicate : Notarchirico à Venosa récemment redaté à 5000 ans $^{6}$, et Cimitero di Atella dont l'attribution chronologique est, en l'état des données, évaluée à environ $600000 \mathrm{ans}^{7}$. La reprise des travaux à Atella représente donc une opportunité unique pour aborder cette question encore largement débattue des processus d'émergence du phénomène bifacial en Europe 8 .

\section{Présentation du site}

Le village d'Atella se trouve dans la province de Potenza en Basilicate, à environ $10 \mathrm{~km}$ au sud du sommet du mont Vulture (fig. 1). Le gisement paléolithique se situe au sud du village actuel, derrière le cimetière municipal moderne, à environ $90 \mathrm{~m}$ au-dessus du niveau actuel du cours d'eau Fiumara di Atella. 
Figure 1 - Localisation du site de Cimitero di Atella.

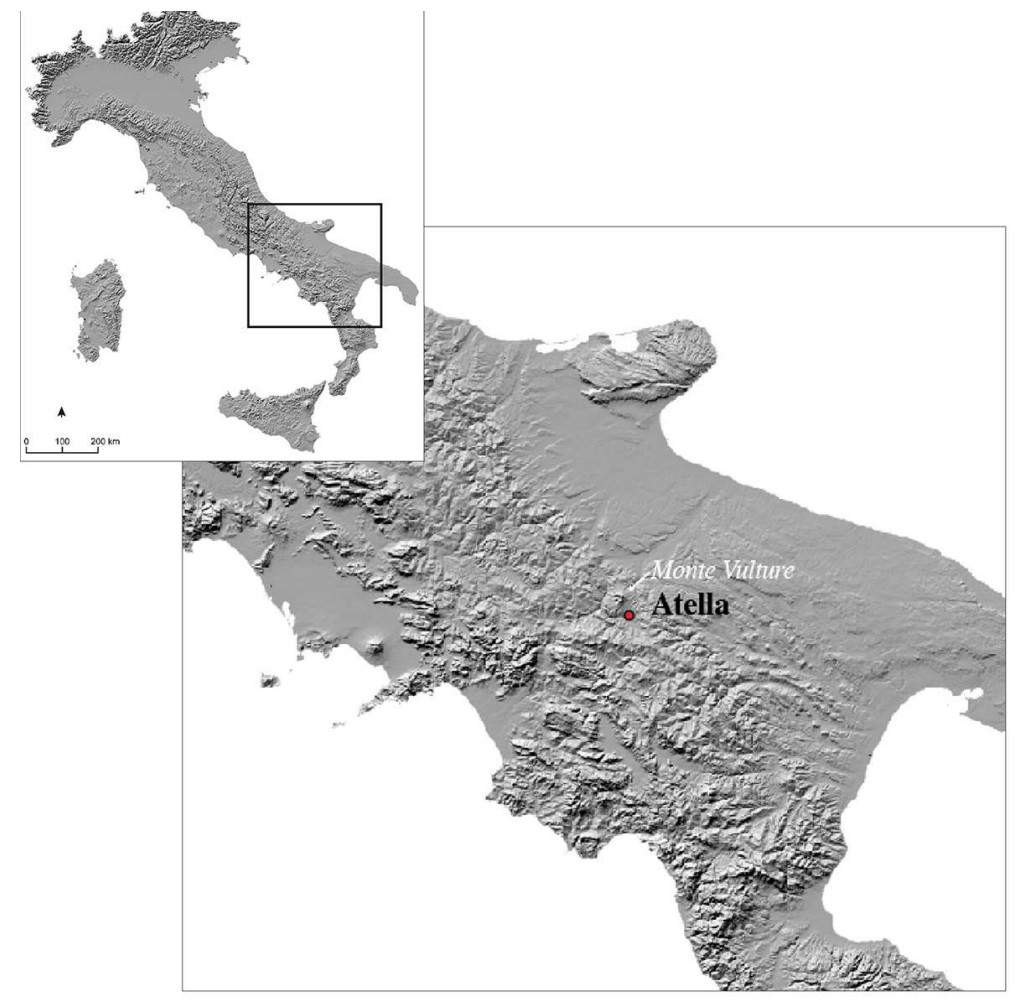

DR

\section{Historique des recherches à Atella}

Le gisement du Cimetière d'Atella a été identifié en 1990, au cours de la mission de fouille d'E. Borzatti et de son équipe sur le site voisin de Masseria Palladino. La fouille, qui a duré plus de 20 ans, concernait une surface d'environ $30 \mathrm{~m}^{2}$, sur une profondeur variable. Le site est visible par le grand public, puisqu'il se trouve couvert par une structure de protection légère (fig. 2). Le gisement a été daté, surtout sur la base de corrélations volcano-stratigraphiques, à environ $600-550 \mathrm{ka}^{9}$. Il semblerait donc que le site de Cimitero di Atella soit légèrement plus récent que Notarchirico ${ }^{10}$ et contemporain d'Isernia-laPineta $^{11}$. Si différents niveaux archéologiques ont été identifiés, le niveau $\mathrm{F}$ a livré la grande majorité du matériel lithique. Le techno-complexe de Cimitero di Atella a été rapporté à l'Acheuléen inférieur sur la base de la présence de «bifaces" au sein de l'outillage ${ }^{12}$. 
Figure 2 - Site d'Atella en 2017.

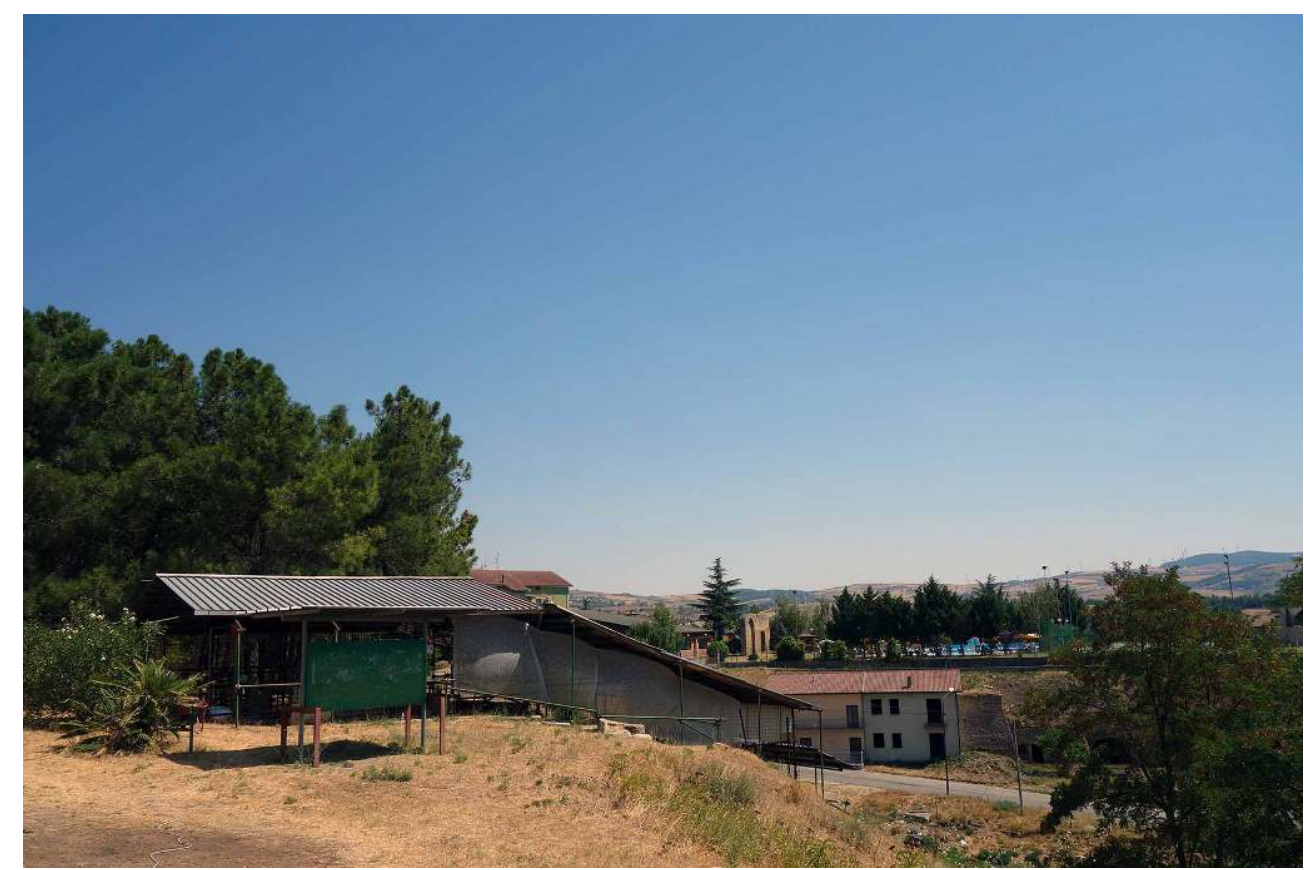

R. Rocca, EFR/SABAP Basilicata.

7 Les restes de faune mis au jour sont principalement représentés par des dents et des fragments de diaphyses d'herbivores. La majorité des restes a été rapportée à Palaeoloxodon antiquus ${ }^{13}$. Il s'agit principalement de deux défenses de morphologie rectiligne, issues de l'ensemble $\mathrm{F}$ à des altitudes différentes, et de molaires. Les autres restes ont été attribués à Bos primigenius ${ }^{14}$ et à différentes espèces de cerfs ${ }^{15}$.

\section{La région du Mont Vulture au Pléistocène moyen : cadre chronologique et environnemental}

Le site d'Atella se trouve dans une région qui a subi de très fortes transformations du paysage depuis le Pléistocène moyen. En effet, le bassin d'Atella a été l'objet d'une forte activité tectonique, par sa position à la croisée des lignes de faille de la fossa bradanica et de la Vulture Line. La région est également marquée par l'importance des activités volcaniques, notamment liées à la proximité avec le mont Vulture. Les recherches sur l'histoire éruptive de ce volcan ${ }^{16}$, sur l'évolution géomorphologique de la région ${ }^{17}$, ainsi que les résultats des travaux autour du site de Notarchirio à Venosa offrent un contexte favorable à l'étude du cadre chronologique des occupations humaines à Atella.

La séquence d'Atella, toujours en cours d'étude, indique la succession de sédiments lacustres, palustres et alluviaux, ce qui représente une opportunité unique pour reconstruire l'évolution du paysage dans le bassin ${ }^{18}$. Si les données issues de la séquence des lacs de Monticchio ${ }^{19}$ ont permis de documenter l'évolution paléo-environnementale au Pléistocène supérieur (130 000-10 000 ans), le Pléistocène moyen (700 000-130 000 ans) est en revanche moins connu, notamment en raison du manque d'archives stratigraphiques disponibles ${ }^{20}$. 


\section{Premiers résultats des campagnes 2016 et 2017}

10 Si la plupart des études sont en cours, les missions d'étude du matériel, les sondages géologiques menés en 2015 et les deux campagnes de fouille 2016 et 2017 ont permis d'apporter des résultats préliminaires sur le gisement d'Atella.

\section{Cadre chronostratigraphique}

11 Concernant le cadre chronostratigraphique, les premières observations permettent de confirmer l'attribution préliminaire qui avait été proposée par E. Borzatti à environ 600 000 ans (de nouvelles analyses chrono-stratigraphiques sont en cours). L'étude de la séquence et des carottages (P. Di Leo, P.Giannandrea, M. Schiattarella) a permis l'enregistrement de quatre grands épisodes survenus dans le bassin d'Atella au cours du Pléistocène moyen :

- Présence d'un lac, dont témoignent plusieurs mètres d'argile à la base de la séquence. Ces niveaux lacustres comprennent des minéraux volcaniques qui sont en cours de datation (A. Pereira et S. Nomade).

- Assèchement progressif du lac et mise en place d'un environnement plus marécageux. C'est au cours de cet épisode qu'a lieu la première fréquentation du site par les groupes humains (outillage en pierre taillée) et animaux (restes de faune, dont une défense d'éléphant).

- Un ou plusieurs épisodes de démantèlement des bordures du lac probablement lié dans une certaine mesure à des épisodes éruptifs du Vulture. Ces colluvions ont conservé des traces du passage de groupes humains et d'animaux (matériel lithique et faunique plus roulé) à proximité du site (ensemble $\mathrm{F}$ de Borzatti). Un niveau de cendre volcanique (tephra) clos cet évènement (étude en cours R. Sulpizio), permettant probablement la meilleure conservation du matériel faunique et lithique observée au sommet de l'ensemble $\mathrm{F}$.

- Mise en place d'un cours d'eau avec des phases de dépôt de sables fins à moyens (datations ESR en cours par P. Voinchet et J.-J. Bahain), entrecoupées d'évènements éruptifs. Cette petite séquence alluviale a également conservé des traces, pour l'instant sporadiques, de fréquentation humaine et animale (ensemble L de Borzatti).

L'élargissement de l'aire de fouille pourrait permettre de trouver dans ces sédiments fins d'autres aires d'occupation mieux conservées.

\section{Réévaluation de l'industrie lithique}

L'industrie lithique mise au jour par E. Borzatti et son équipe entre 1990 et 2010 est issue des deux principaux ensembles identifiés, les niveaux L et F. La majeure partie du matériel appartenant à l'ensemble $\mathrm{F}$ a été attribuée à l'Acheuléen inférieur sur la base de la présence d'un outillage lourd sur radiolarite poreuse (bifaces et pebble-tools) et d'un outillage léger de petite dimension en silex (encoches, denticulés et racloirs) ${ }^{21}$. Contrairement à celui de l'ensemble $\mathrm{F}$, le matériel lithique issu des niveaux $\mathrm{L}$ ne comprend pas de bifaces, mais uniquement la composante dite légère ${ }^{22}$.

13 Une nouvelle étude technologique menée sur le matériel des niveaux $\mathrm{F}^{23}$ a permis d'apporter de nouveaux éléments sur la collection issue des anciennes fouilles et de 
nuancer certaines interprétations. La première étape a consisté à trier le matériel en excluant de l'étude d'une part les pièces sans attribution stratigraphique claire et d'autre part les éléments non anthropiques ou douteux. Ainsi, sur les plus de 20000 pièces qui formaient initialement la collection, 638 ont été étudiées. Trois chaînes opératoires principales ont été identifiées. Les indices d'une chaîne opératoire de confection de «petits outils $~^{24}$ sont bien représentés sous la forme des outils même (194 pièces) (fig. 3) ainsi que de petits éclats d'encoches et de retouches caractéristiques. Une chaîne opératoire de débitage a également été identifiée, les 13 nucléus ainsi qu'une partie des éclats sont compatibles avec des systèmes de production additionnels ${ }^{25}$ qui visent dans ce cas à l'obtention d'éclats courts souvent à dos. Le nombre des « macro-outils » découverts en contexte stratigraphique dans le niveau $\mathrm{F}$ a été considérablement réduit. Ces pièces, précédemment interprétées comme des bifaces, ont été réalisées dans une matière première qui s'est altérée (probablement en relation avec la desilicification d'un calcaire fin) rendant difficile leur lecture (fig. 4). Le support sélectionné est plano-convexe ; il s'agit souvent d'un grand éclat ou d'une portion de galet, et le façonnage concerne généralement une seule face mais jamais tout le volume du support. Plusieurs types de tranchant ont été aménagés sur ces pièces, qui devront être comparées avec les autres pièces découvertes lors des missions 2016 et 2017.

Figure 3 - Cimitero di Atella, petit outil en silex, niveau F (fouille E. Borzatti).

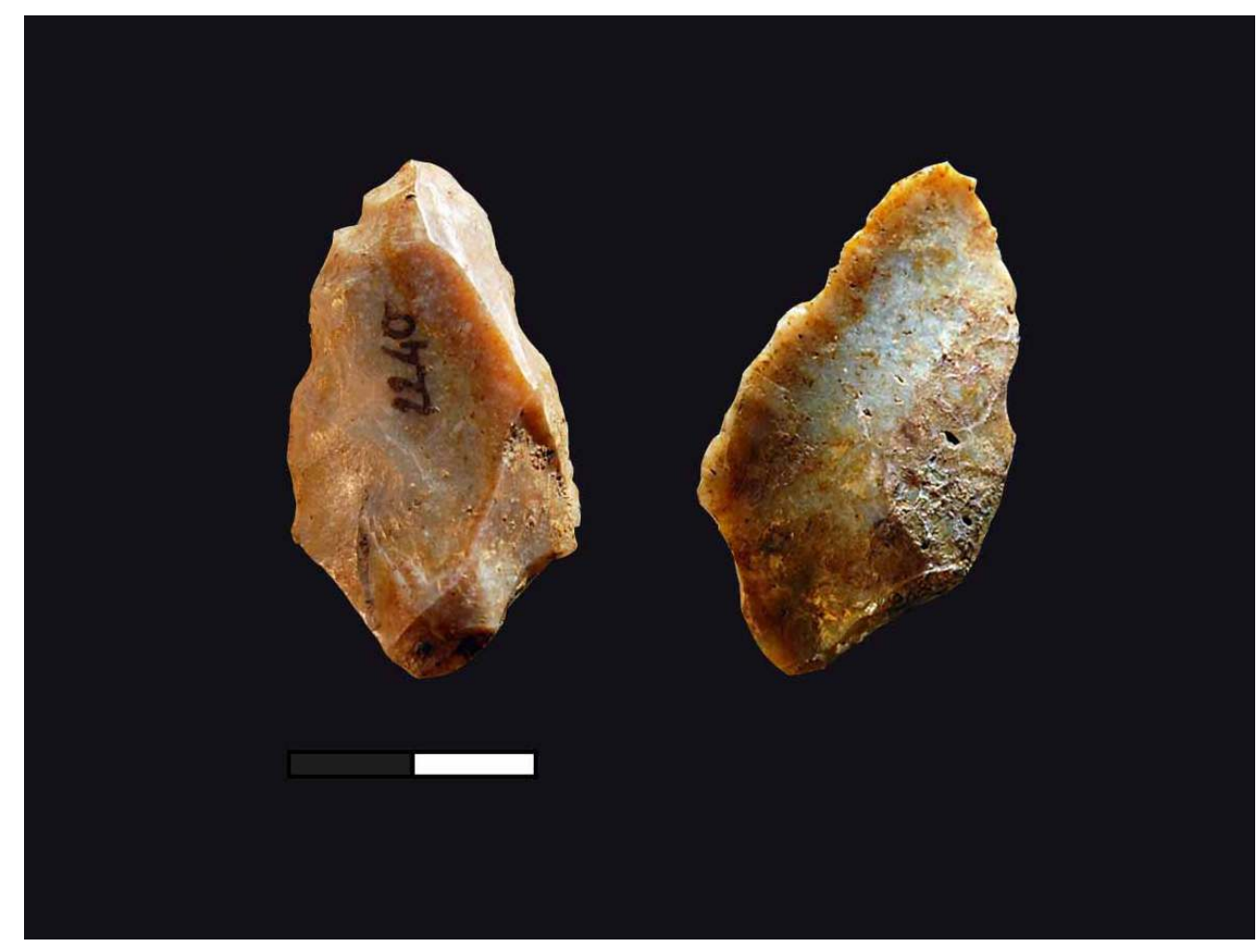

R. Rocca, EFR/SABAP Basilicata. 
Figure 4 - Cimitero di Atella, macro-outil en calcaire très altéré, niveau F (fouille E. Borzatti).

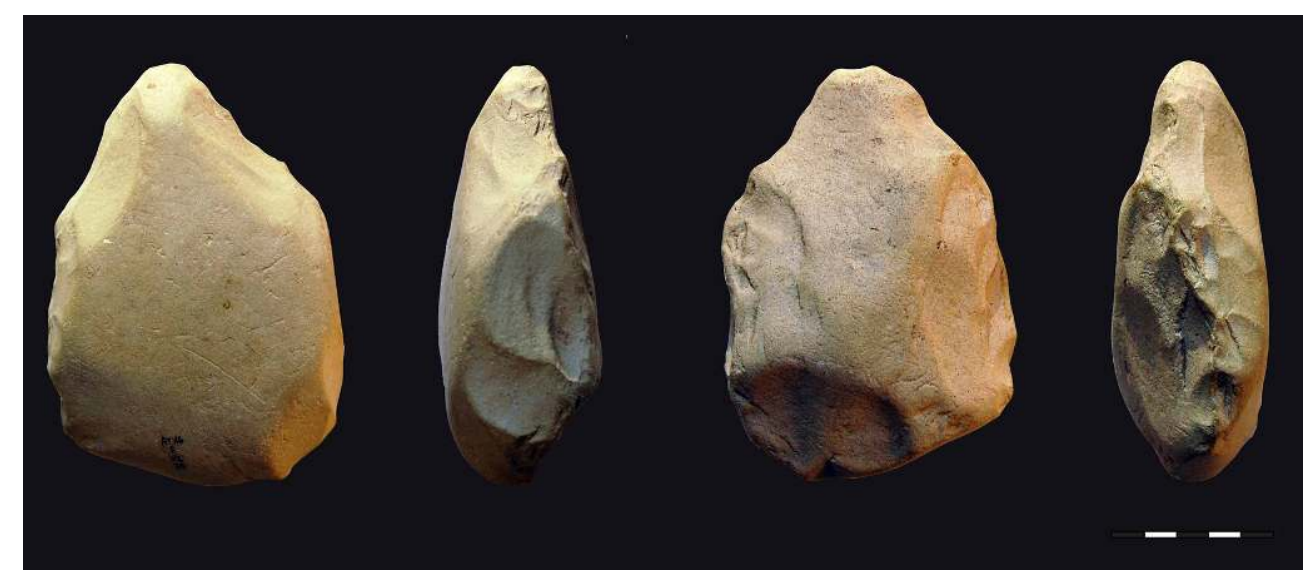

R. Rocca, EFR/SABAP Basilicata.

14 Cette étude des niveaux $\mathrm{F}$ a permis d'apporter de nouveaux éléments à l'analyse des processus de formation de cet ensemble. En effet, les états de surface altérés des pièces et la présence d'éléments naturels grossiers indiquent clairement que le matériel lithique a été remanié et déposé en position secondaire lors d'un ou de plusieurs épisodes de colluvionnement. Toutefois, le matériel comprend des indices cohérents de trois chaines opératoires - débitage d'éclats, confection de petits outils et façonnage de rares grands outils -, caractères attendus pour cette période marquée par l'émergence balbutiante du phénomène bifacial.

15 Concernant le matériel lithique de l'ensemble L (fouille Borzatti), l'étude préliminaire a permis de confirmer l'absence de macro-outillage. La collection, relativement peu riche, comporte principalement des petits outils, ainsi que des éclats probablement issus de leur chaîne opératoire de confection.

Les pièces mises au jour durant les campagnes 2016 et 2017 appartiennent également aux chaînes opératoires identifiées dans les anciennes collections. Le matériel, toujours en cours d'étude, comprend principalement des petits éclats en silex issus de la confection de petits outils. Les grands outils façonnés demeurent rares, tout comme les éclats de façonnage et les indices de chaîne opératoire de débitage. Ces premières observations sur les industries lithiques d'Atella sont cohérentes avec ce que l'on connaît des systèmes techniques de cette période en Europe méditerranéenne. En effet, outre la présence de rares bifaces, le débitage d'éclats et la confection de petits outils semblent représenter une part importante des assemblages de nombreux sites entre 800000 et 400000 dans le sud de l'Europe ${ }^{26}$.

\section{Réévaluation de la faune}

Les restes de faune mis au jour par E. Borzatti et son équipe ont principalement été attribués à Palaeoloxodon antiquus, mais également à Bos primigenius et à plusieurs espèces de cervidés (Dama, Cervus et Capreolus) ${ }^{27}$. La plupart des restes ont été découverts dans l'ensemble F, l'ensemble L n'ayant livré que peu de restes et aucun attribuable au Palaeoloxodon (fig. 5) ${ }^{28}$. 
Figure 5 - Cimitero di Atella, défense de Palaeoloxodon, mission 2017.

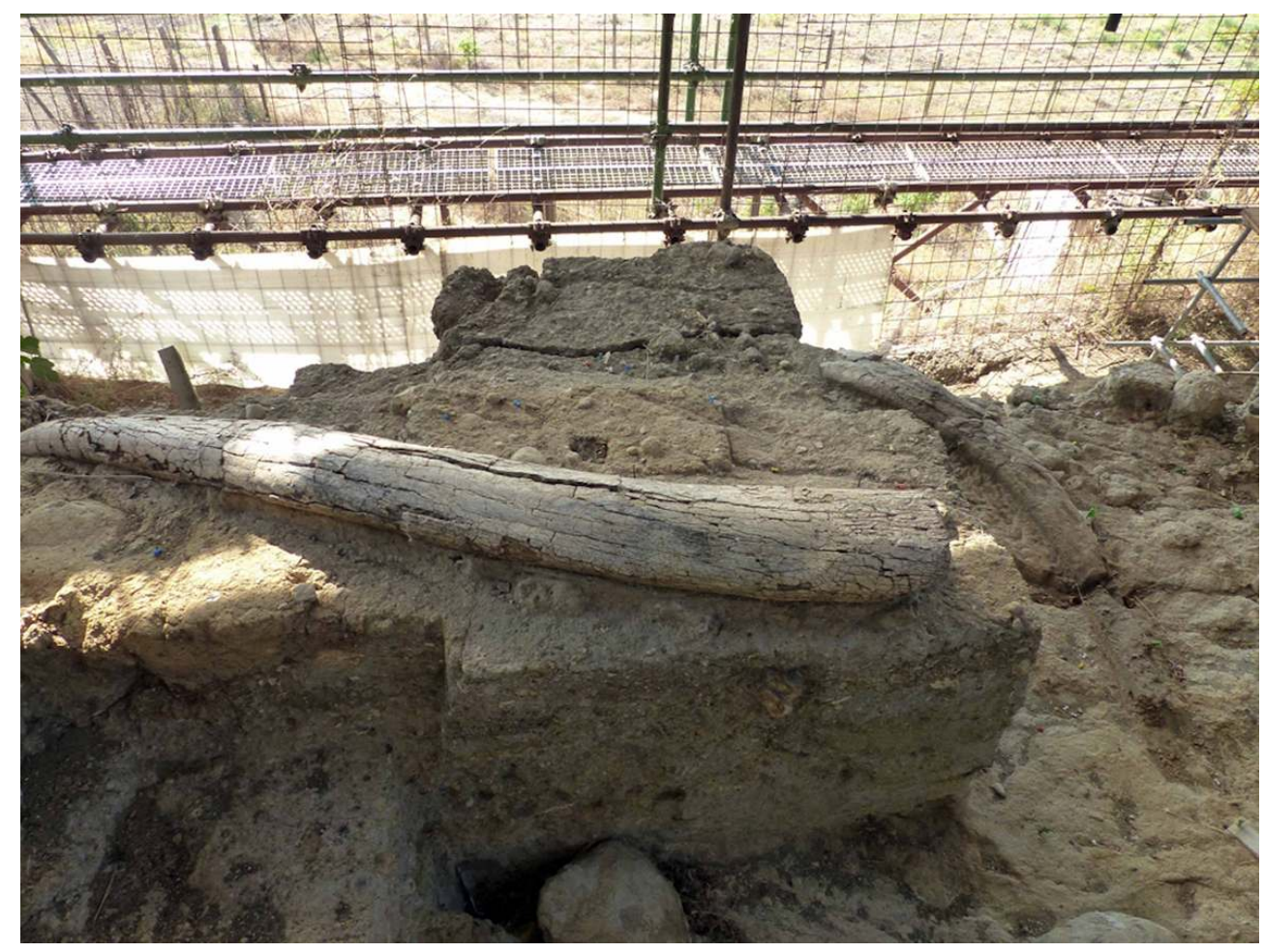

R. Rocca, EFR/SABAP Basilicata.

18 Les anciennes collections et le matériel des nouvelles fouilles sont encore en cours d'étude (R. Sardella pour la paléontologie et F. Boschin pour l'archéozoologie). Les observations préliminaires indiquent que le matériel est très fragmenté et contient donc peu de restes déterminables tandis que l'état de conservation apparaît très variable au sein d'un même ensemble. La présence de Palaeoloxodon, de cervidés, de daims et de bovidés est confirmée, même si la distinction entre Bos et Bison est encore à démontrer. Par contre, aucun reste de chevreuil n'a été identifié et on note également l'absence des autres espèces typiques de tels assemblages, comme les chevaux et les sangliers. Les carnivores et petits animaux (types lagomorphes) sont également absents à ce jour. Les modalités de fouille, de restauration et de conditionnement précédentes ont probablement nuit à la bonne conservation des faunes. Nous espérons que les nouvelles stratégies mises en place depuis 2016 permettront d'augmenter le degré de restes déterminables et de mener des analyses taphonomiques sur la faune.

\section{Perspectives}

\section{Dynamique de formation du site}

Si les analyses visant à replacer le site dans son contexte chronostratigraphique large sont en cours de publication, il s'agit désormais de s'interroger sur les processus de formation des dépôts à une échelle plus fine. Les stratégies de fouilles mises en œuvre lors des campagnes de l'ensemble $F$ (pendage et orientation des pièces, tamisage, etc.) ainsi que les prélèvements micro-morphologiques (analyses M. Zambaldi) réalisés en 2017 devront permettre de préciser la dynamique de formation du dépôt. En effet, les 
premières observations stratigraphiques semblent indiquer que l'ensemble $\mathrm{F}$ serait le résultat d'un ou de plusieurs processus de colluvionnement complexes, dont il convient de reconstituer la rythmicité et l'intensité afin de pourvoir évaluer l'histoire taphonomique du matériel archéologique.

\section{Système technique à Atella}

Si l'analyse des anciennes collections a permis de réévaluer les chaînes opératoires représentées à Atella, il est désormais nécessaire d'ouvrir de nouveaux secteurs de fouille en adaptant la stratégie à l'histoire taphonomique de chacun des ensembles. Si une partie du matériel est issue de dépôts perturbés (ensemble F), il convient d'en évaluer l'ampleur et d'analyser le matériel en conséquence. Toutefois certains secteurs, encore évalués de façon trop préliminaire, apparaissent prometteurs en termes de conservation (ensemble L, sommet et base du F) et permettront peut-être d'apporter des informations d'une autre nature (spatiales, techno-économiques, subsistances, etc.).

L'analyse des nouvelles collections qui devraient s'enrichir dans les années à venir permettra vraisemblablement de préciser la structuration des chaînes opératoires. Quelle est la place des grands outils façonnés dans l'assemblage? Comment comparer ces pièces aux autres macro-outils qui apparaissent un peu partout en Europe au cours de cette phase chronologique? Quelle diversité technique peut-on identifier sur les petits outils en termes fonctionnels? Plusieurs questions subsistent, elles devront être envisagées à partir du matériel d'Atella, mais aussi en dialogue avec les résultats des très nombreuses études menées sur cette période en Italie et dans le nord de la Méditerranée.

\section{Paysage et environnement}

22 La séquence d'Atella nous offre une fenêtre sur l'évolution du paysage au Pléistocène moyen dans la région. Les collaborations en cours sur l'évolution géomorphologique (P.Giannadrea et M. Schiattarella), paléoclimatique (P.Di Leo) mais aussi sur la paléontologie (R. Sardella) et la paléobotanique (D. Magri et F. Di Rita) contribuent à apporter de nouvelles connaissances sur le territoire et sur l'environnement fréquenté par les hommes il y a 600000 ans.

\section{Valorisation}

La structure de métal recouverte de tôles ondulées, qui entoure jusqu'à aujourd'hui le site, apparaît inadaptée à la conservation des dépôts et du matériel archéologique, au bon déroulement des recherches scientifiques et à la visite du site par le grand public. Un projet de muséalisation du site a été conçu en collaboration avec les institutions locales et nationales (Soprintendenza Archeologia Belle Arti e Paesaggio della Basilicata, commune d'Atella, région Basilicate) afin d'assurer à long terme la poursuite des travaux scientifiques et de la valorisation du gisement d'Atella (Abruzzese et al. 2016).

\section{Conclusion}

Les résultats préliminaires des recherches menées sur le site de Cimitero Atella ont confirmé l'importance du site dans le cadre des problématiques sur les dynamiques de 
peuplement au Paléolithique ancien dans le nord de la Méditerranée. Les nouvelles études sur les collections mises au jour par la fouille d'E. Borzatti, la reprise des travaux de terrain et la mise en place de nouvelles collaborations afin de replacer le site dans son contexte chronostratigraphique et environnemental ont confirmé la présence de plusieurs occupations humaines à Atella il y a 600000 ans, moment clé dans l'histoire des dynamiques de peuplement en Europe. La poursuite des travaux de terrain - qui serait facilitée par la mise en place d'une nouvelle structure de protection du site -, ainsi que la finalisation des analyses en cours permettront de préciser les modalités de ces occupations et d'affiner la connaissance sur les systèmes techniques de ces groupes humains ainsi que sur les stratégies d'interaction avec leur milieu.

\section{BIBLIOGRAPHIE}

Abruzzese 2014 = C. Abruzzese, Il sito paleolitico di Atella (PZ) nel quadro del primo popolamento europeo: studio tecnologico dell'industria litica, Tesi di Laurea Magistrale, Università di Napoli «L'Orientale», Naples, 2014.

Abruzzese - Aureli - Rocca 2016 = C. Abruzzese, D. Aureli, R. Rocca, Assessment of the Acheulean in southern Italy: New study on the Atella site (Basilicata, Italy), dans Quaternary International, 393, 2016, p. 158-168.

Allen - Watts - Huntley 2000 = J.R.M. Allen, W.A. Watts, B. Huntley, Weichselian palynostratigraphy, palaeovegetation and palaeoenvironment; the record from Lago Grande di Monticchio, southern Italy, dans Quaternary International, 73-74, 2000, p. 91-110.

Arzarello - Peretto 2010 = M. Arzarello, C. Peretto, Out of Africa: The first evidence of Italian peninsula occupation, dans Quaternary International, 223-224, 2010, p. 65-70.

Arzarello - De Weyer - Peretto 2016 = M. Arzarello, L. De Weyer, C. Peretto, The first European peopling and the Italian case: Peculiarities and “opportunism", dans Quaternary International, 393, 2016, p. 41-50.

Aureli et al. 2016 = D. Aureli, R. Rocca, C. Lemorini, V. Modesti, S. Scaramucci, S. Milli, B. Giaccio, F. Marano, M.R. Palombo, A. Contardi, Mode 1 or mode 2? "Small tools" in the technical variability of the European Lower Palaeolithic: The site of Ficoncella (Tarquinia, Lazio, central Italy), dans Quaternary International, 393, 2016, p. 169-184.

Bar-Yosef - Belfer-Cohen 2001 = O. Bar-Yosef, A. Belfer-Cohen, From Africa to Eurasia - early dispersals, dans Quaternary International, 75-1, 2001, p. 19-28.

Barsky - de Lumley 2010 = D. Barsky, H. de Lumley, Early European Mode 2 and the stone industry from the Caune de l'Arago's archeostratigraphical levels "P", dans Quaternary International, 223-224, 2010, p. 71-86.

Boëda 2005 = É. Boëda, Paléo-technologie ou anthropologie des techniques, dans Arob@se, 1, 2005, p. 46-64. 
Boëda 2013 = É. Boëda, Techno-logique \& technologie, 2013.

Bonadonna et al. 1998 = F.P. Bonadonna, D. Brocchini, M.A. Laurenzi, C. Principe, G. Ferrara, Stratigraphical and chronological correlations between Monte Vulture volcanics and sedimentary deposits of the Venosa Basin, dans Quaternary International, 47, 1998, p. 87-96.

Borzatti von Löwenstern 1998 = E. Borzatti von Löwenstern, Il Bacino di Atella nella Preistoria, dans Studi per l'ecologia del quaternario, 20, 1998, p. 7-39.

Borzatti von Löwenstern 2005 = E. Borzatti von Löwenstern, Il sito Acheuleano antico del Cimitero di Atella: gli strati alti del sedimento preistorico, dans Studi per l'ecologia del quaternario, 27, 2005.

Borzatti von Löwenstern et al. 1990 = E. Borzatti von Löwenstern, M. Sozzi, S. Vannucci, F. Vianello, L'acheuleano antico del cimitero di Atella, dans Prime indagini sulla stratigrafia del sedimento e sulle industrie litiche, in studi per l'ecologia del quaternario, 12, 1990, p. 9-30.

Borzatti von Löwenstern et al. 1997 = E. Borzatti von Löwenstern, A. Palchetti, M. Sozzi, M. Maestrini, Témoignages de l'Acheuléen inférieur en Italie méridionale : Le gisement du Cimitero di Atella (Basilicata), dans L'Anthropologie, 101-4, 1997, p. 617-638.

Brocchini et al. 1994 = D. Brocchini, L. La Volpe, M.A. Laurenzi, C. Principe, Storia evolutiva del Monte Vulture, dans Plinius, 12, 1994, p. 22-25.

Carbonell et al. 1999 = E. Carbonell, M. Mosquera, X.P. Rodríguez, R. Sala, J. van der Made, Out of Africa: The dispersal of the Earliest technical systems eeconsidered, dans Journal of Anthropological Archaeology, 18-2, 1999, p. 119-136.

Carbonell - Rodríguez 2006 = E. Carbonell, X.P. Rodríguez, The first human settlement of Mediterranean Europe, dans Comptes Rendus Palevol, 5, 2006, p. 291-298.

Ciolli $1997=$ N. Ciolli, Elephas antiquus Falconer \& Cautley del Cimitero di Atella (Pz), dans Studi per l'ecologia del quaternario, 19, 1997, p. 25-34.

Combourieu-Nebout et al. $2015=$ N. Combourieu-Nebout, A. Bertini, E. Russo-Ermolli, O. Peyron, S. Klotz, V. Montade, S. Fauquette, J. Allen, F. Fusco, S. Goring, B. Huntley, S. Joannin, V. Lebreton, D. Magri, E. Martinetto, R. Orain, L. Sadori, Climate changes in the central Mediterranean and Italian vegetation dynamics since the Pliocene, dans Review of Palaeobotany and Palynology, 218, 2015, p. 127-147.

Di Leo et al. 2011 = P. Di Leo, D. Gioia, C. Martino, A. Pappalardo, M. Schiattarella, Geomorphological, mineralogical, and geochemical evidence of Pleistocene weathering conditions in the Southern Italian Apennines, dans Geologica Carpathica, 62-1, 2011, p. 43-53.

Di Muro 1999 = A. Di Muro, Inquadramento tefrostratigrafico del sito acheuleano del Cimitero di Atella (Basilicata-Italia), dans Studi per l'ecologia del quaternario, 21, 1999, p. 7-14.

Gallotti 2016 = R. Gallotti, The East African origin of the Western European Acheulean technology: Fact or paradigm?, dans Quaternary International, 411, 2016, p. 9-24.

Gallotti - Peretto 2015 = R. Gallotti, C. Peretto, The Lower/early Middle Pleistocene small débitage productions in Western Europe: New data from Isernia La Pineta t. 3c (Upper Volturno Basin, Italy), dans Quaternary International, 357, 2015, p. 264-281.

García-Medrano et al. 2014 = P. García-Medrano, A. Ollé, M. Mosquera, I. Cáceres, C. Díez, E. Carbonell, The earliest Acheulean technology at Atapuerca (Burgos, Spain): Oldest levels of the Galería site (GII Unit), dans Quaternary International, 353, 2014, p. 170-194. 
Giannandrea et al. 2006 = P. Giannandrea, L. La Volpe, C. Principe, M. Schiattarella, Unità stratigrafiche a limiti inconformi e storia evolutiva del vulcano medio-pleistocenico di Monte Vulture (Appennino meridionale, Italia), dans Bollettino-Società Geologica Italiana, 125-1, 2006, p. 67.

Moncel $2010=$ M.-H. Moncel, Oldest human expansions in Eurasia: Favouring and limiting factors, dans Quaternary International, 223-224, 2010, p. 1-9.

Moncel - Schreve 2016 = M. Moncel, D. Schreve, The Acheulean in Europe: Origins, evolution and dispersal, dans Quaternary International, 411, 2016, p. 1-8.

Moncel et al. 2013 = M.-H. Moncel, J. Despriée, P. Voinchet, H. Tissoux, D. Moreno, J.-J. Bahain, G. Courcimault, C. Falguères, Early evidence of Acheulean settlement in Northwestern Europe - La Noira Site, a 700000 year-old occupation in the Center of France, dans PLOS ONE, 8-11, 2013, p. e75529.

Moncel et al. sous-presse = M.-H. Moncel, M. Arzarello, É. Boëda, S. Bonilauri, B. Chevrier, C. Gaillard, H. Forestier, L. Yinghua, F. Sémah, V. Zeitoun, The assemblages with bifacial tools in Eurasia (first part). What is going on in the West? Data on western and southern Europe and the Levant, dans Comptes Rendus Palevol.

Mosquera et al. 2016 = M. Mosquera, A. Ollé, P. Saladié, I. Cáceres, R. Huguet, A. Rosas, J. Villalaín, A. Carrancho, D. Bourlès, R. Braucher, A. Pineda, J. Vallverdú, The Early Acheulean technology of Barranc de la Boella (Catalonia, Spain), dans Quaternary International, 393, 2016, p. 95-111.

Mosquera et al. 2018 = M. Mosquera, A. Ollé, X.P. Rodríguez-Álvarez, E. Carbonell, Shedding light on the Early Pleistocene of TD6 (Gran Dolina, Atapuerca, Spain): The technological sequence and occupational inferences, dans PLOS ONE, 13-1, 2018, p. e0190889.

Nicoud 2013 = É. Nicoud, Complexité des stratigraphies régionales et nouveau modèle d'apparition et de diffusion du phénomène Acheuléen en Europe de l'ouest, dans Quaternaire. Revue de l'Association française pour l'étude du Quaternaire, 24-4, 2013, p. 407-417.

Nicoud et al. 2016 = E. Nicoud, D. Aureli, M. Pagli, V. Villa, C. Chaussé, S. Agostini, J.-J. Bahain, G. Boschian, J.-P. Degeai, F. Fusco, B. Giaccio, M. Hernandez, C. Kuzucuoglu, C. Lahaye, C. Lemorini, N. Limondin-Lozouet, P. Mazza, N. Mercier, S. Nomade, A. Pereira, V. Robert, M.A. Rossi, C. Virmoux, A. Zupancich, Preliminary data from Valle Giumentina Pleistocene site (Abruzzo, Central Italy): A new approach to a Clactonian and Acheulian sequence, dans Quaternary International, 409, 2016, p. 182-194.

Parfitt et al. 2005 = S.A. Parfitt, R.W. Barendregt, M. Breda, I. Candy, M.J. Collins, G.R. Coope, P. Durbidge, M.H. Field, J.R. Lee, A.M. Lister, R. Mutch, K.E.H. Penkman, R.C. Preece, J. Rose, C.B. Stringer, R. Symmons, J.E. Whittaker, J.J. Wymer, A.J. Stuart, The earliest record of human activity in Northern Europe, dans Nature, 438, 2005, p. 1008-1012.

Pereira 2017 = A. Pereira, Apport de la datation 40Ar/39Ar à la compréhension de l'évolution culturelle des pré-néanderthaliens en Italie centrale et méridionale entre 750 et $250 \mathrm{ka}$, thèse de doctorat, Muséum national d'histoire naturelle de Paris, Université de Ferrare, Paris-Ferrare, 2017.

Pereira et al. 2015 = A. Pereira, S. Nomade, P. Voinchet, J.-J. Bahain, C. Falguères, H. Garon, D. Lefèvre, J.-P. Raynal, V. Scao, M. Piperno, The earliest securely dated hominin fossil in Italy and evidence of Acheulian occupation during glacial MIS 16 at Notarchirico (Venosa, Basilicata, Italy), dans Journal of Quaternary Science, 30-7, 2015, p. 639-650.

Peretto et al. 2015 = C. Peretto, J. Arnaud, J. Moggi-Cecchi, G. Manzi, S. Nomade, A. Pereira, C. Falguères, J.-J. Bahain, D. Grimaud-Hervé, C. Berto, B. Sala, G. Lembo, B. Muttillo, R. Gallotti, U.T. Hohenstein, C. Vaccaro, M. Coltorti, M. Arzarello, A human deciduous tooth and new 40Ar/39Ar dating results from the middle pleistocene archaeological site of Isernia La Pineta, Southern Italy, dans PLOS ONE, 10-10, 2015, p. e0140091. 
Peretto et al. 2016 = C. Peretto, M. Arzarello, J.-J. Bahain, N. Boulbes, J.-M. Dolo, E. Douville, C. Falguères, N. Frank, T. Garcia, G. Lembo, A.-M. Moigne, B. Muttillo, S. Nomade, A. Pereira, M.A. Rufo, B. Sala, Q. Shao, U. Thun Hohenstein, U. Tessari, M.C. Turrini, C. Vaccaro, The Middle Pleistocene site of Guado San Nicola (Monteroduni, Central Italy) on the Lower/Middle Palaeolithic transition, dans Quaternary International, 411, 2016, p. 301-315.

Piperno 1999 = M. Piperno (éd.), Notarchirico: un sito del Pleistocene medio antico nel bacino di Venosa, Venosa, 1999.

Rocca 2016 = R. Rocca, Depuis l'Est ? Nouvelles perspectives sur les premières dynamiques de peuplement en Europe, dans L'Anthropologie, 120-3, 2016, p. 209-236.

Rocca - Abruzzese - Aureli 2016 = R. Rocca, C. Abruzzese, D. Aureli, European Acheuleans: Critical perspectives from the East, dans Quaternary International, 411, 2016, p. 402-411.

Rocca et al. 2016 = R. Rocca, C. Abruzzese, D. Aureli, J.-J. Bahain, P. Beneduce, P. Di Leo, P. Giannandrea, S.I. Giano, D. Gioia, D. Magri, S. Nomade, A. Pereira, M. Schiattarella, R. Sulpizio, P. Voinchet, New multidisciplinary research project on the Lower Palaeolithic site of Cimitero di Atella (Potenza), dans F. Negrino, F. Fontana, A. Moroni, J. Riel-Salvatore (éd.), The Palaeolithic and Mesolithic in Italy: new research and perspectives DAFIST, Genoa (Italy) - 4th-5th February 2016, Florence, 2016, p. 21-22.

Santagata $2016=$ C. Santagata, Operating systems in units B and E of the Notarchirico (Basilicata, Italy) ancient Acheulean open-air site and the role of raw materials, dans Quaternary International, 411, 2016, p. 284-300.

Schiattarella et al. 2005 = M. Schiattarella, P. Beneduce, P. Di Leo, S.I. Giano, P. Giannandrea, C. Principe, Assetto strutturale ed evoluzione morfotettonica quaternaria del vulcano del Monte Vulture (Appennino Lucano), dans Bollettino-Società Geologica Italiana, 124-3, 2005, p. 543.

Sharon - Barsky 2016 = G. Sharon, D. Barsky, The emergence of the Acheulian in Europe - A look from the east, dans Quaternary International, 411, 2016, p. 25-33.

Villa - Buettner 2009 = I.M. Villa, A. Buettner, Chronostratigraphy of Monte Vulture volcano (southern Italy): secondary mineral microtextures and 39Ar-40Ar systematics, dans Bull Volcanol, 71-10, 2009, p. 1195.

Villa et al. 2016 = P. Villa, S. Soriano, R. Grün, F. Marra, S. Nomade, A. Pereira, G. Boschian, L. Pollarolo, F. Fang, J.-J. Bahain, The Acheulian and Early Middle Paleolithic in Latium (Italy): Stability and Innovation, dans PLOS ONE, 11-8, 2016, p. e0160516.

Watts - Allen - Huntley 1996 = W.A. Watts, J.R.M. Allen, B. Huntley, Vegetation history and palaeoclimate of the last glacial period at Lago Grande di Monticchio, Southern Italy, dans Quaternary Science Reviews, 15-2, 1996, p. 133-153.

Wulf et al. 2012 = S. Wulf, J. Keller, M. Paterne, J. Mingram, S. Lauterbach, S. Opitz, G. Sottili, B. Giaccio, P.G. Albert, C. Satow, others, The 100-133 ka record of Italian explosive volcanism and revised tephrochronology of Lago Grande di Monticchio, dans Quaternary Science Reviews, 58, 2012, p. 104-123.

Zucchelli $1999=$ M. Zucchelli, Bos primigenius Bojanus del Bacino di Atella (Potenza), dans Studi per l'ecologia del quaternario, 21, 1999, p. 19-30.

Zucchelli $2000=$ M. Zucchelli, Nuovi ritrovamenti di resti osteologici di Bos primigenius Bojanus nel Bacino di Atella (Potenza), dans Studi per l'ecologia del quaternario, 22, 2000, p. 11-22.

Zucchelli $2002=$ M. Zucchelli, I cervidi dell'Acheuleano antico del Cimitero di Atella (Potenza), dans Studi per l'ecologia del quaternario, 24, 2002, p. 3-17. 


\section{NOTES}

1. Bar-Yosef - Belfer-Cohen 2001 ; Carbonnel et al. 1999 ; Moncel 2010.

2. Arzarello - Peretto 2010 ; Carbonell - Rodríguez 2006 ; Parfitt et al. 2005.

3. Rocca 2016.

4. Arzarello - De Weyer - Peretto 2016 ; Aureli et al. 2016 ; Gallotti - Peretto 2015 ; Nicoud et al. 2016 ; Peretto et al. 2016 ; Villa et al. 2016.

5. García-Medrano et al. 2014 ; Moncel et al. 2013 ; Mosquera et al. 2016 ; Piperno 1999.

6. Pereira et al. 2015.

7. Borzatti von Löwenstern et al. 1997.

8. Boëda 2005; Gallotti 2016; Moncel et al. sous-presse ; Moncel - Schreve 2016; Nicoud 2013 ; Rocca - Abruzzese - Aureli 2016 ; Sharon - Barsky 2016.

9. Borzatti von Löwenstern 1998 ; Di Muro 1999

10. Pereira et al. 2015 ; Piperno 1999.

11. Peretto et al. 2015.

12. Borzatti von Löwenstern et al. 1990 ; Borzatti von Löwenstern et al. 1997.

13. Ciolli 1997.

14. Zucchelli 1999 ; Zucchelli 2000.

15. Zucchelli 2002.

16. Bonadonna et al. 1998 ; Brocchini et al. 1994 ; Villa - Buettner 2009.

17. Giannandrea et al. 2006 ; Schiattarella et al. 2005.

18. Rocca et al. 2016

19. Watts - Allen - Huntley 1996 ; Allen - Watts - Huntley 2000 ; Di Leo et al. 2011 ; Wulf et al. 2012.

20. Combourieu-Nebout et al. 2015.

21. Borzatti von Löwenstern et al. 1997.

22. Borzatti von Löwenstern 2005.

23. Abruzzese 2014 ; Abruzzese - Aureli - Rocca 2016.

24. Aureli et al. 2016 ; Rocca - Abruzzese - Aureli 2016 ; Rocca 2016.

25. Boëda 2013.

26. Barsky - de Lumley 2010 ; Gallotti - Peretto 2015 ; Mosquera et al. 2018 ; Rocca - Abruzzese Aureli 2016 ; Santagata 2016.

27. Borzatti et al. 1997 ; Zucchelli 1999 ; Zucchelli 2000 ; Zucchelli 2000 ;

28. Borzatti 2005.

\section{RÉSUMÉS}

Le site de Cimitero di Atella, découvert et fouillé pendant plus de vingt ans par E. Borzatti et son équipe, a livré les indices de plusieurs occupations attribuées au Paléolithique ancien (environ 600000 ans). Les précédentes études menées sur le matériel lithique ont conduit à attribuer ces 
occupations à l'Acheuléen ancien, sur la base de la présence de biface, outil qui émerge en Europe justement à cette période pour perdurer durant tout le Paléolithique ancien (jusqu'à 300000 ans environ). La reprise des travaux à Atella, dans le cadre d'un nouveau projet de recherche de l'École française de Rome sur les dynamiques de peuplement au Paléolithique en Italie (programme quinquennal PALEO) a pour objectif de poursuivre les activités de terrain, de réétudier le matériel lithique et faunique, et de protéger et valoriser le gisement. En 2016 et 2017, les missions d'étude et de fouille nous ont conduit à nous interroger sur la place d'Atella dans le panorama du Paléolithique ancien en Méditerranée. La poursuite des activités permettra de préciser le cadre chronostratigraphique ainsi que les processus de formation du site.

Cimitero di Atella, discovered and excavated for 20 year by E. Borzatti and his team, yielded evidence of several Lower Palaeolithic occupations (circa 600,000 years ago). The previous research on the lithic assemblage lead to the attribution of these occupations to Lower Acheulean because of the presence of Handaxes. This tool emergence properly at this period in Europe and will last up to the end of Lower Palaeolithic (circa 300,000 years ago). The resumption of activities at Atella is part of a new project of the French School of Rome on the settlement dynamic during the Palaeolithic in Italy (quinquennial program PALEO). The research at Atella focus on fieldwork, lithic and faunal remains study and protection and enhancement of the site. In 2016 and 2017, excavation and study lead us to question the role of Atella in the Mediterranean Lower Palaeolithic framework. Long-term work studies will allow also to precise the chronostratigraphic context and the site formation processes.

\section{INDEX}

Index géographique : Atella

Keywords : Lower Palaeolithic, basilicata, lithic industry, Italy, Atella

Mots-clés : Paléolithique ancien, industrie lithique, Italie, Basilicate, Atella

\section{AUTEURS}

\section{ROXANE ROCCA}

Université Paris 1 Panthéon-Sorbonne - UMR 7041 ArScan ethnologie préhistorique, roxane.rocca@univ-paris1.fr

\section{DANIELE AURELI}

Università di Bologna, Università di Siena - UMR 7041 ArScan, AnTET, daniele.aureli@unibo.it

\section{CLAUDIA ABRUZZESE}

Università degli studi di Napoli «L'Orientale», cla.abruzzese@gmail.com 